\title{
A Review of the Experience with Pediatric Written Requests Issued for Oncology Drug Products
}

\author{
Alemayehu Akalu ${ }^{1}$ Xi Meng², Gregory Reaman ${ }^{3}$, Lian Ma ${ }^{1}$, Weishi Yuan ${ }^{4}$, and Jingjing \\ $\mathrm{Ye}^{5}$ \\ ${ }^{1}$ US Food and Drug Administration \\ ${ }^{2}$ University of Massachusetts Amherst School of Public Health and Health Sciences \\ ${ }^{3}$ Children's National Health System \\ ${ }^{4}$ Imab \\ ${ }^{5}$ BeiGene US
}

September 16, 2020

\begin{abstract}
Background Pediatric anticancer drug development has numerous challenges. The PREA and the BPCA were passed to address the deficiency in pediatric drug development in general. Until recently, the requirement for pediatric evaluation of most oncology products has been waived, because children typically do not have the same type of cancers which occur commonly in adults or the indication or drug had been granted orphan designation. PREA therefore had no impact. Pediatric studies for labeling updates are largely done through BPCA by a Written Request, issued by FDA. Because pediatric and adult populations do not share the same biology, natural history, or disease progression, there are limited opportunities to extrapolate adult efficacy and safety to the pediatric population. The requirements for the pediatric studies have varied greatly over time. Procedure In this study, we searched WRs that were issued by the FDA since 2001. We found 40 such requests issued for oncology drugs and biologics which had been accepted by sponsors. Results Clinical trials included in 23 of the WRs have been concluded, 19 have resulted in exclusivity, and 3 drugs that were studied have been approved for use in pediatric populations. Herein we present the spectrum of WRs from a regulatory, study design, dosing, formulation, analysis plan, evidential standard of efficacy and safety perspective. Conclusions This provides information on requests in the past nearly 20 years and studies completed. As WRs are the anchor of pediatric cancer development for the time being, this can potentially provide insight on how pediatric cancer drug development may change in the future.
\end{abstract}

\section{A Review of the Experience with Pediatric Written Requests Issued for Oncology Drug Prod- ucts}

Alemayehu Y. Akalu, PharmD ${ }^{\wedge}$, Xi Meng ${ }^{\wedge}$, M.S., Gregory H. Reaman ${ }^{1,5^{*}}$, MD, Lian Ma ${ }^{3}$, Ph.D., Weishi Yuan $^{4 \#}$, Ph.D., Jingjing Ye ${ }^{4 *}$, Ph.D.

${ }^{1}$ Office of Oncologic Diseases, Office of New Drugs, Center for Drug Evaluation and Research, Food and Drug Administration, Silver Spring, MD, USA; ${ }^{2}$ Department of Biostatistics, School of Public Health \& Health Sciences, University of Massachusetts-Amherst; ${ }^{3}$ Division of Pharmacometrics (DPM), Office of Clinical Pharmacology (OCP); ${ }^{4}$ Division of Biostatistics IX (DBIX), Office of Biostatistics (OB), Office of Translational Sciences (OTS), Center for Drug Evaluation and Research (CDER), Food and Drug Administration (FDA); ${ }^{5}$ Oncology Center of Excellence (OCE), Food and Drug Administration, Silver Spring, MD, USA

\#Completed while work at FDA, ^ co-first author, ${ }^{*}$ co-corresponding author: Gregory H. Reaman, 10903 New Hampshire Ave, Silver Spring, MD, 20993. (301)796-0785. Gregory.Reaman@fda.hhs.gov ; and Jingjing Ye, 8170 Maple Lawn Blvd, Suite 160, Fulton, MD. (628)239-0505, jingjingye@gmail.com. 


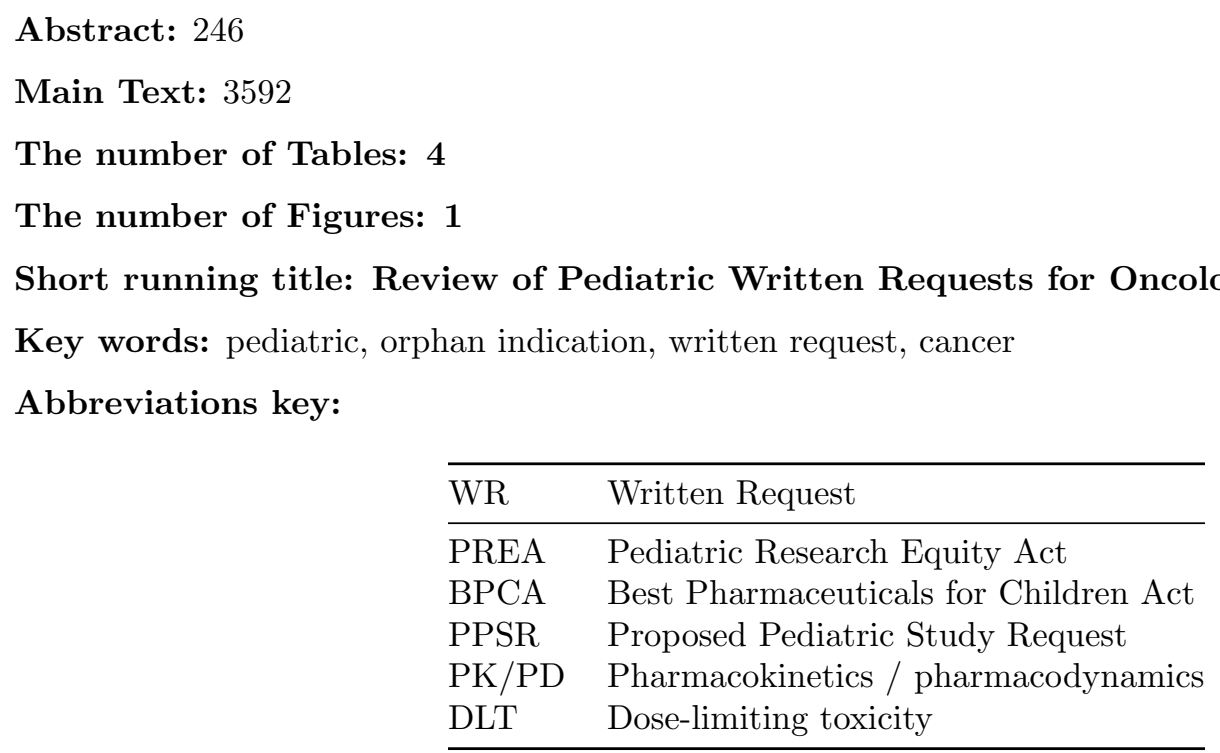

Disclaimer: The opinions expressed are those of the authors, and do not reflect the position of the U.S. Food and Drug Administration.

\section{Abstract:}

\section{Background}

Pediatric anticancer drug development has numerous challenges. The Pediatric Research Equity Act (PREA) and the Best Pharmaceuticals for Children Act (BPCA) were passed to address the deficiency in pediatric drug development in general. Until recently, the requirement for pediatric evaluation of most oncology products developed for adult cancers has been waived, because children typically do not have the same type of cancers which occur commonly in adults or the indication or drug had been granted orphan designation. PREA therefore had no impact. Pediatric studies for labeling updates are largely done through BPCA by a Written Request (WR), issued by FDA. Because pediatric and adult populations do not share the same biology, natural history, or disease progression, there are limited opportunities to extrapolate adult efficacy and safety to the pediatric population. The requirements for the pediatric studies have varied greatly over time.

\section{Procedure}

In this study, we searched WRs that were issued by the FDA since 2001. We found 40 such requests issued for oncology drugs and biologics which had been accepted by sponsors.

\section{Results}

Clinical trials included in 23 of the WRs have been concluded, 19 have resulted in exclusivity, and 3 drugs that were studied have been approved for use in pediatric populations. Herein we present the spectrum of WRs from a regulatory, study design, dosing, formulation, analysis plan, evidential standard of efficacy and safety perspective.

\section{Conclusions}

This provides information on requests in the past nearly 20 years and studies completed. As WRs are the anchor of pediatric cancer development for the time being, this can potentially provide insight on how pediatric cancer drug development may change in the future.

Key words: pediatric, orphan indication, written request, cancer 


\section{Introduction}

A 1968 editorial article in the Journal of Pediatrics referred to children as "therapeutic orphans" [1] to express the frustration of many clinicians over the lack of pediatric prescribing information for approved drugs [2]. For the past 5 decades, pediatric cancer drug development has faced biological, societal and economic challenges, due to low prevalence patient population, concerns related to ethical issues and perception of increased liability in testing drugs in children, and lack of financial incentives.

Two laws passed by the U.S. Congress, the Pediatric Research Equity Act (PREA) [3], which provides the requirement for pediatric studies, and the Best Pharmaceuticals for Children Act (BPCA) [4], which provides the incentive of additional exclusivity for products of sponsors who voluntarily conduct requested studies in the pediatric population, were enacted in 2003 and 2002 respectively, following the initial legislative provision for exclusivity in 1997 to correct this serious deficiency in drug development for young patients.

The requirement under PREA for pediatric evaluation of most oncology products developed for adult cancers is generally waived, because the common cancers which occur in adults and which are the focus of drug discovery and development efforts are never or very rarely seen in children or because the indication or drug had been granted orphan designation. PREA therefore has had no impact on pediatric anticancer drug development. Pediatric trials for labeling updates are largely done through BPCA by the fulfillment of a Written Request (WR), issued by the FDA. Because cancers in the pediatric and adult populations generally do not share the same biology, natural history and disease progression, full extrapolation from adults is unlikely, and requirements for the pediatric trials can vary greatly according to the disease indications. As a result, the requirements of oncology drug trials in WR vary greatly. As BPCA has been the only legislative initiative that impacts pediatric cancer drug development, more recent WRs were issued for products early in development to accelerate pediatric assessment and requirements for studies included in the WRs were contingent upon the results of a preceding study.

The objective of this research is to identify, review, and evaluate all the written requests (WRs) for pediatric clinical trials of new drug products for solid tumor and hematologic malignancies that were initially issued by the Food and Drug Administration between the dates January 1, 2001, and December 31, 2019. The authors from the Office of Biostatistics (OB), Office of Clinical Pharmacology (OCP) in CDER and Oncology Center of Excellence (OCE) have collaborated to review the content of WRs issued to sponsors for oncology drugs and biologics.

\section{Methods}

\section{Selection of Studies}

The information that has been summarized in this paper was gathered from public information about the WRs that were issued by the FDA between the dates of January 1, 2001, and December 31, 2019 in response to Proposed Pediatric Study Requests (PPSRs). When the trials were completed and final clinical summary reports submitted in response to WRs and made available (https://www.fda.gov/drugs/developmentresources/written-requests-issued, https://www.fda.gov/drugs/development-resources/list-determinationsincluding-written-request and https://www.fda.gov/media/79154/download), a comprehensive survey was conducted on the trial results and corresponding FDA reviews, as well as published papers related to the pediatric studies. We excluded those pediatric WRs submitted to the National Institute of Child Health and Human Development, National Institutes of Health (NIH) for off patent drugs.

\section{Technical Information}

For each WR, key information included, but was not limited to specific pediatric cancer indications, feasibility of extrapolation from adults trials, number of studies requested, number of cohorts, designs, and phases of the studies (phase 1/2 dose-escalation/dose-finding, signal of activity seeking, proof of concept phase 2 studies or phase 3), study drugs (monotherapy, combination), study designs (single-arm or with control, any blinding, dose levels and more), age eligibility, study endpoints, number and reasons for amendments. For the trials submitted to fulfill the WR, key information was captured, including patient accrual in trials 
included the WR, statistical methods used for efficacy evaluation, primary results, dose levels, starting dose selection, pharmacokinetics (PK) / pharmacodynamics (PD) sampling and analysis, unique dose-limiting toxicity (DLT) observed in children and special formulations developed for children. Finally, the current status of the WR was summarized, and whether or not pediatric exclusivity was granted.

A single WR generally contains more than one trial and more than one cohort/indication, since the WRs are expected to address all possible pediatric indications for which the investigational drug might provide a public health benefit be included in the assessment of the drug. Because labeling updates and regulatory actions are taken for an individual drug, therefore, the results were summarized for each WR rather than individual trials in the WR. The number of patients included in each WR was obtained by summing the numbers of trial subjects among all trials and cohorts/indications included in the WR. If any one of the trials has a control/comparator arm, the WR is categorized as controlled. If any one of the trials in WR employed monotherapy, the WR is categorized as monotherapy.

In the trials, the primary and secondary endpoints, were grouped into the following types: objective response rate endpoints, including overall response rate $(\mathrm{ORR})$, complete response rate $(\mathrm{CR})$, and complete remission (CR), which are binary; time to event (TTE) endpoints, including overall survival (OS), disease-free survival (DFS), Event-free survival (EFS) and progression-free survival (PFS); dose finding endpoints, including determination of maximum tolerated dose (MTD), and recommended phase 2 dose (RP2D); PK/PD endpoints; safety endpoints; and any other endpoints, including change from baseline and unspecified or exploratory efficacy endpoints.

Completed WRs are those where complete study report from the WR studies were submitted to the FDA in the form of product supplement or labeling revision as a sNDA/BLA. The completed WRs were categorized as such if any of the following were met: exclusivity granted, denied exclusivity, under review, completed but fail to submit reports according to timeline required in the written requests, and completed but no remaining exclusivity. In contrast, WRs were considered as "not completed" if any of the following were met: terminated, listed as withdrawal/released, or categorized as ongoing.

Unique DLTs are defined as DLTs that are either only found or found with increased incidence in pediatric patients.

Because children differ from adult including capacities for drug administration, medicine-related toxicity and taste preference, different formulations for children may be required and the WR may include special formulation development. This includes extemporaneous compounding of marketed adult formulations for investigational use or a required marketable formulation.

\section{Results}

\section{Overall/Regulatory}

During the 19-year period, the FDA issued 40 WRs to study drug products for solid tumors and/or hematologic malignancies in the pediatric population. Of the studies included in $40 \mathrm{WRs}, 27$ were for pediatric solid tumors, 18 were for pediatric hematologic malignancy, and 5 of the WRs included studies for both solid tumor and hematologic malignancies. The WRs that were identified include pediatric oncology trials conducted in pediatric patients from the neonatal period up to the age of 17. A few of the clinical trials included young adults up to the age of 30 . The list of drugs for which WRs were issued, the initial request dates, current status of each WR, the approved age range and sections related to WRs included in the labeling are shown in Table 1. The table includes the information that is either listed from https://www.fda.gov/drugs/developmentresources/list-determinations-including-written-request or https://www.fda.gov/media/79154/download or additional websites are included as footnotes in the table.

Fifteen out of 27 WRs that included solid tumor studies and 11 out of 18 WRs that included hematologic malignancies studies have been completed (23 out of $40 \mathrm{WRs}$ completed in total, and 3 of the WRs included studies for both solid tumor and hematologic malignancies). Ninety-two percent (21/23) of the completed WRs submitted reports to the FDA in accordance with the timeline required in written requests. Three 
oncology drugs for the treatment of solid tumor or hematologic malignancies resulted in approval of the product for pediatric use added in the US prescribing information (USPI) specifically for pediatric age groups.

Of the completed WRs, 14 out of 15 WRs that include studies for solid tumors and 5 out of 11 WRs that include studies for hematologic malignancies have been granted 6 -month exclusivity. One WR was denied exclusivity because of insufficient patients enrolled to assess the efficacy or for insufficient ability to inform a description of pharmacokinetic $(\mathrm{PK})$ parameters. One pediatric trial was terminated or released due to enrollment difficulty ( $1 \mathrm{WR}$ ). Two WRs were completed; however, the timeline to submit had passed. Finally, 1 drug has no remaining patent life. Therefore, there is no exclusivity to add. A summary of pediatric exclusivity status for the completed WRs is shown in Table 2.

Requests for amendments to the WRs were submitted to the FDA in 53\% (21 WRs) of the 40 and 20\% (8 WRs) were amended upon request from the sponsor more than twice. The reasons for requests to amend a Written Request include a change in age distribution reflecting accrual expectations in practice, a cancelation of planned studies, an updated timeline, an increase or decrease in disease cohorts, and adjustment to the study endpoints (e.g. adding/removing a safety or efficacy endpoint and replacing MTD with RP2D). Other less common reasons for requesting amendments include a change of dose levels, a change of therapy, a change of formulations, and a change of statistical evaluation method or criteria.

TABLE 1: List of Issued Written Requests (WRs) between Jan. 1, 2001 and Dec. 31, 2019 (selected with public information)

TABLE 2: Summary of pediatric exclusivity status for the completed WRs

FIGURE 1. The distribution of disease cohorts for WRs and the number of patients enrolled for completed pediatric trials. The upper left panel (1a) shows the number of disease cohorts in solid tumor studies, the upper right (1b) shows that for studies of hematologic malignancies. The $\mathrm{x}$ axis represents the number of disease cohorts, and the y axis shows the number of WRs.

Among the pediatric trials initiated since 2001 in $40 \mathrm{WRs}$, extrapolation from adult trials was planned or used to support efficacy in pediatrics for 5 drugs, given similar underlying pathobiology and mechanism of action in pediatric patients and adults.

\section{Study Designs}

The study designs in this section are summarized based on amended WRs rather than original WRs if the final study reports submitted were for trials conducted according to amended WRs. The solid tumor trials in 24 out of 27 WRs included both phase 1 and 2 studies. The trials in 2 WRs only included phase 2 studies because the maximum tolerated dose (MTD) or recommended phase 2 dose (RP2D) in pediatrics had already established from trials conducted previously. Trials in 3 WRs were designed to have a tentative phase 2 study based on results from the phase 1 study. For those WRs that include trials for hematologic malignancies 12 out of $18 \mathrm{WRs}$ included both phase 1 and 2 studies, trials in 2 WRs included only a phase 1 study, and trials in 4 WRs included a phase 2 study only.

For solid tumors, trials in 14 WRs have a control arm, with 3 used placebo with either best supportive care (BSC) or standard of care (SOC) and the rest 11 included active control arms. The other trials in 13 WRs are designed as single-arm. As a comparison, for hematologic malignancies, majority (15 out of 18) was single-arm design and only 3 have an active control.

As for treatment arm, trials in $23 \mathrm{WRs}$ for solid tumor and $13 \mathrm{WRs}$ for hematologic malignancies used monotherapy as the treatment arm; trials in $4 \mathrm{WRs}$ for solid tumor and $5 \mathrm{WRs}$ for hematologic malignancies used combination therapies.

\section{Study Endpoints}

For efficacy endpoints, trials in $30 \mathrm{WRs}$ used a response rate, a binary endpoint, as the primary endpoint. 
This includes trials in 25 WRs used overall response rate (ORR) assessed by investigators, 2 used overall response rate assessed by independent review committees (IRC), 5 used a complete response (CR) rate assessed by investigators and 1 used a complete response rate assessed by an IRC. Some trials have both ORR and CR rates evaluations. Pediatric trials in 5 WRs used the time to event as efficacy endpoint, with 2 using event-free survival (EFS), 1 progression-free survival (PFS) assessed by investigators, and 2 used progression-free survival (PFS) assessed by an IRC.

Within one WR, typically a dose-escalation/dose-determination phase/study is required to evaluate the PK, safety, and preliminary activity of the drug to establish the MTD and/or RP2D in pediatric patients. For programs where the extrapolation from adult efficacy was feasible, the dose-finding phase/study was to identify the optimal safe and tolerable dose in pediatrics that achieves similar exposures to those achieved in adults. Other studies require different primary endpoints: 18 pediatric trials included PK/PD endpoints, 14 pediatric trials used safety related primary endpoint and 11 used other primary endpoints include clinical activity such as a change from baseline or other unspecified efficacy or activity endpoints.

Commonly used secondary endpoints include response rate, time to event endpoints, PK/PD endpoints, safety endpoints, and many other endpoints such as palatability and electrocardiogram measures.

\section{Analysis and Sample Size}

A minimum number of patients is typically required for individual study. We compared the number of patients required in the WR vs the number actually enrolled to fulfill the WR when the WR is completed. Eight pediatric trials in WRs for solid tumors and 2 pediatric trials in WRs for hematologic malignancies recruited less patients than requested; while 3 pediatric trial for solid tumor and 3 pediatric trials for hematologic malignancies recruited more patients than requested.

\section{Safety Evaluation}

Unique dose limiting toxicity (DLT) that are either only found or found with increased incidence in pediatric patients was observed in several drug (TABLE 3).

Irinotecan $\mathrm{HCl}$ (Camptosar) has reported different adverse event profile in pediatric patients from adults in that dehydration associated with severe hypokalemia and hyponatremia (Section 8.4 of USPI [20]). Nilotinib (Tasigna) reported to have observed growth retardation (Section 5.14 in USPI [11]). Sunitinib malate (Sutent) has reported dose-limiting cardiotoxicity, which prompted amendment of the study to exclude patients with previous exposure to anthracyclines or cardiac radiation (Section 8.4 of USPI [13]). Capecitabine (Xeloda) has reported more frequently laboratory abnormalities. For details, refer to section 8.4 of USPI [15].

TABLE 3. Unique DLTs observed in oncology pediatric trials in response to written requests initiated since 2001.

\section{Efficacy Evaluation}

Efficacy endpoints used for supporting approvals in WRs include overall response rate (ORR), complete response (CR), event-free survival (EFS), and progression-free survival (PFS).

Efficacy evaluation methods that have been used are Cochran-Mantel-Haenszel test for response rate endpoints, Kaplan-Meier curve for time to event endpoints, t-test, and analysis of covariance (ANCOVA) for change from baseline endpoints.

Trials in nineteen WRs were designed to be powered to evaluate the primary efficacy. Among those 19 WRS, $12 \mathrm{WRs}$ are considered completed. In the actual results submitted in response to WRs, only seven were able to conduct hypothesis testing in actual trials. Main reasons for not able to conduct hypothesis testing are due to present efficacy results descriptively.

\section{PK/PD Evaluation}


Pharmacokinetics and/or pharmacodynamics evaluation was required in all WRs and listed as primary endpoint in 18 pediatric trials. In all competed or ongoing studies in response to the WRs, PK samples were collected through rich or sparse sampling to be analyzed with Population PK analysis and/or noncompartmental PK analysis. Key PK parameters (AUC, Cmax, Clearance, and volume of distribution, etc) were obtained. Depending on the planned age range for each study, PK collection was required from a minimum of 6-10 patients in each of the following age groups: 1 month to $<2$ years or 12 months to $<6$ years, 6 to $<12$ years, and 12 to $<18$ years of age. Using combined data from available studies, Population PK/PD modeling and/or exposure-response analysis for safety and efficacy were conducted or planned to determine the optimal dose in the target pediatric population. In recent WRs for large molecules, characterization of immunogenicity was also required.

\section{Starting dose Selection}

The information for starting dose in phase 1 pediatrics trials was obtained for 37 WRs. The approaches for starting dose selection generally fall in two categories: empirical, and PK modeling and simulation. A summary of methods used for each approach by disease type is shown in Table 4. Majority of the program used the empirical approach of selecting a starting dose equivalent to $100 \%$ (most common for hematological malignancy) or $\sim 80 \%$ (most common for solid tumor) of body size adjusted adult dose, while 7 programs used $50-70 \%$ of adult MTD or RP2D to initial their phase 1 dosing-finding studies. As a comparison, PK modeling and simulation was used to identify the starting dose in pediatric patients for 7 drugs. This approach typically includes allometric scaling of adult PK parameters (i.e., clearance and volume of distribution) based on body weight in children, and account for developmental factors such as organ and enzyme maturation where ontogeny functions are added for the dose projection. For one program, non-clinical data in murine model was also used to inform the starting dose selection for a combination therapy, along with data from earlier monotherapy study in pediatrics.

TABLE 4: Methods for starting dose selection in pediatric oncology studies in response to written requests initiated since 2001.

\section{Formulation}

The development of an age-appropriate formulation and commitment to commercialization if use of the product is extended to the pediatric population based on study results is a requirement of the WR. Bioavailability of any formulation used in the studies must be characterized, and as needed, a relative bioavailability study comparing the approved drug to the age appropriate formulation may be conducted in adults. Special formulations have been developed for 5 drugs in response to the WRs initiated since 2001. For example, the adult formulation of capecitabine is tablets. Pediatric film-coated tablets were designed to quickly disperse in water so that can be administered to pediatric patients who are unable to swallow tablets. (https://www.fda.gov/media/87706/download).

\section{Discussion}

Thoughtful drug development and timely assessment of promising novel agents in pediatric patients is critical to public health. Over the years, pediatric cancer drug development has relied solely on BPCA to advance drug development and inclusion of pediatric information in the drug label. FDA has issued 40 Written Requests for oncology products during the study period. In recent years some of these Written Requests were issued to sponsors early in the drug development timeline and before approval. Since BPCA and the Written Request mechanism was the only legislative initiative available to facilitate pediatric investigations, the FDA has attempted to maximize its authority to issue Written Requests as early as possible in a product's development when there was interest in the pediatric cancer academic and advocacy communities to evaluate the potential activity of certain promising agents. Nineteen drugs have been granted pediatric exclusivity, and 3 received approval for use in specific age groups with the indication(s) in the drug label. Most oncology pediatric trials have been designed to evaluate optimal dose, PK, and both preliminary safety and efficacy. The majority of pediatric trials in response to WRs included evaluation of dose and dose-limiting toxicities of the agent being studied and monotherapy activity. More recent Written Requests that included studies 
beyond phase 1or phase 2 evaluations were contingent upon data from initial studies demonstrating safety and signal of activity with plans for definitive studies subject to review of and concurrence with protocols by the Agency prior to study initiation. For this reason, most pediatric trials in the WRs are single arm design, especially for hematologic malignancies. Although controlled trials are preferred, they are not feasible to conduct within the context of a Written Request because of the small number of study subjects and the time to completion of complex, randomized studies. Most efficacy endpoints involved overall response rate as assessed by investigators; some studies required response rates to be determined by an independent review committee (IRC).

More pediatric trials are still urgently needed. Accrual challenges remain. Many of the completed pediatric trials over the past 19 years have led to conclusions that the cancer drugs developed for adult cancer indications have not demonstrated sufficient effectiveness within the context of limited phase 1 and/or 2 studies in heavily pre-treated patients.

Because of many challenges in pediatric cancer drug development, innovative approaches should be explored to improve the efficiency of pediatric trials. Since children are a vulnerable population, it is challenging to enroll them in clinical trials where the potential for beneficial effects of investigational drugs may be unclear. Given the uncertainty in drug efficacy and for the protection of pediatric patients, the implementation of futility criteria in the trial design should be considered in future pediatric trials. Trials should stop early if evidence of no efficacy is observed to minimize the number of patients exposed to ineffective therapies. A Bayesian approach is a flexible tool that could be used in pediatric trials to sequentially monitor efficacy and futility as data accumulate [37]. This approach provides an option to stop trials for efficacy or futility if enough evidence is observed, and thus with the advantages of possibly requiring fewer patients and shorter trials. Bayesian approaches in pediatric trials can formally incorporate prior information from adults, older age groups, and other external sources if appropriate and quantify the uncertainty. To overcome the limitation of a small population and limited opportunities for extrapolation, an innovative approach using Bayesian strategy to allow more flexibility in statistical design for future pediatric trials should be considered.

Identifying an optimal starting dose remains important for successful pediatric oncology drug development. Modeling and simulation are a powerful tool to inform selection of safe and efficacious doses as early as possible in the drug development process and to avoid overexposing children to subtherapeutic doses. When the disease pathobiology and the mechanism of action for drug would not differ by age, a starting dose may be selected by targeting similar exposure to the adult therapeutic dose without the need to evaluate multiple dose levels. When similarity in disease between pediatric and adults cannot be assumed, a more extensive PK and dose finding study may be required. While traditional designs like the $3+3$ and rolling 6 are commonly used for dose escalation, Bayesian designs like the continual reassessment method or adaptive logistic regression design could be preferable, especially for targeted therapies with safety profiles well characterized in adults.

Under the FDA Reauthorization Act (FDARA) of 2017 which incorporates the RACE for Children Act [35], pediatric trials for oncology products will no longer be exempt based on infeasibility related to the adult indication for which the drug is being developed or orphan designation. Early pediatric assessment of dose, tolerability and signal of activity will be required for drugs directed at molecular targets observed in pediatric cancers [36]. Original marketing applications in the US for certain adult oncology drugs that are submitted on or after August 18, 2020, will be required to be preceded by plans for pediatric cancer investigations. Thus, pediatric drug development will finally be coordinated with cancer drug development for adults, as part of an overall drug development plan.

We conducted this review prior to the implementation of FDARA Sec. 504 on the Written Requests issued over a 19-year period to demonstrate differences in requirements and the variable response to those requirements. Overall, this report may serve to guide the planning of future cancer drug development for children and adolescents.

\section{Conflict of Interest}

The authors have no conflicts of interest to disclose. JY is currently an employee of BeiGene Ltd, WY is 
currently an employee of IMab and the work on this manuscript was completed while employed at FDA.

\section{Acknowledgements}

\section{Data Availability Statement}

Data sharing is not applicable to this article as no new data were created in this study.

\section{References}

1. Shirkey H. Editorial comment: Therapeutic orphans. The Journal of Pediatrics, 1968: 72(1): 119-120.

2. Institute of Medicine. Safe and Effective Medicines for Children: Pediatric Studies Conducted Under the Best Pharmaceuticals for Children Act and the Pediatric Research Equity Act. Washington DC: The National Academies Press. https://doi.org/10.17226/13311; 2012.

3. PREA. Pediatric Research Equity Act (PREA). Retrieved from https://www.ncbi.nlm.nih.gov/books/NBK3997/

4. BPCA. Best Pharmaceuticals for Children's Act (BPCA). Retrieved from https://bpca.nichd.nih.gov/Pages/default.aspx

5. Yondelis. USPI. Retrieved from https://www.accessdata.fda.gov/drugsatfda_docs/label/2018/207953s005lbl.pdf

6. Abraxane. USPI. Retrieved from https://www.accessdata.fda.gov/drugsatfda_docs/label/2019/021660s046lbl.pdf

7. Jevtana. USPI. Retrieved from https://www.accessdata.fda.gov/drugsatfda_docs/label/2020/201023s023lbl.pdf

8. Velcade. USPI. Retrieved from https://www.accessdata.fda.gov/drugsatfda_docs/label/2019/021602s044lbl.pdf

9. Afinitor. USPI. Retrieved from https://www.accessdata.fda.gov/drugsatfda_docs/label/2020/022334s044,203985s016lbl.pdf

10. Treanda. USPI. Retrieved from docs/label/2019/022249s024lbl.pdf

11. Tasigna. USPI. Retrieved from docs/label/2019/022068s031lbl.pdf

12. Taxotere. USPI. Retrieved from docs/label/2019/020449s083lbl.pdf

13. Sutent. USPI. Retrieved from docs/label/2019/021938s036lbl.pdf

14. Methotrexate. USP. Retrieved from https://www.accessdata.fda.gov/drugsatfda_docs/label/2018/011719s125lbl.pdf

15. Xeloda. USPI. Retrieved from docs/label/2019/020896Orig1s042lbl.pdf

16. Eloxatin. USPI. Retrieved from docs/label/2020/021759s023lbl.pdf

17. Zometa. USPI. Retrieved from docs/label/2018/021223s041lbl.pdf

18. phosphate. USPI. Retrieved from docs/label/2011/022137s003lbl.pdf

19. Arimidex. USPI. Retrieved from docs/label/2018/020541s031lbl.pdf

20. Camptosar. USPI. Retrieved from docs/label/2020/020571s051lbl.pdf 21. Temodar. USPI. Retrieved from https://www.accessdata.fda.gov/drugsatfda_docs/label/2019/022277s013lbl.pdf

22. Navelbine. USPI. Retrieved from docs/label/2020/020388s037lbl.pdf

https://www.accessdata.fda.gov/drugsatfda_-

https://www.accessdata.fda.gov/drugsatfda_-

https://www.accessdata.fda.gov/drugsatfda_-

https://www.accessdata.fda.gov/drugsatfda_-

https://www.accessdata.fda.gov/drugsatfda_-

https://www.accessdata.fda.gov/drugsatfda_-

https://www.accessdata.fda.gov/drugsatfda_-

https://www.accessdata.fda.gov/drugsatfda_-

https://www.accessdata.fda.gov/drugsatfda_-

https://www.accessdata.fda.gov/drugsatfda_https://www.accessdata.fda.gov/drugsatfda_- 
23. Gemzar. USPI. Retrieved from https://www.accessdata.fda.gov/drugsatfda_docs/label/2019/020509s082lbl.pdf

24. Honig, S. 2002. Medical review for Navelbine (vinorelbine). NDA 20388/S014. Nov. 2002. Silver Spring, MD: Food and Drug Administration. http://www.accessdata.fda.gov/drugsatfda_docs/nda/2002/020388_S014_NAVELBINE_INJECTION_AP.pdf (accessed April 3, 2012)

25. Gemzar (gemcitabine HCL) pediatric written request. Jan. 2001. Silver Spring, MD: Food and Drug Administration. https://www.fda.gov/media/88726/download

26. Pediatric Exclusivity Granted, Apr. 2016. Silver Spring, MD: Food and Drug Administration. https://www.fda.gov/media/79154/download

27. Shapiro, A. 2003. Medical review for Temodar (Teozolomide). NDA 21029/S005. Mar. 2003. Silver Spring, MD: Food and Drug Administration. https://www.fda.gov/media/92216/download

28. Ibrahim, A. 2003. Medical review for Camptosar (Irinotecan). NDA 20571/S021. Dec. 2003. Silver Spring, MD: Food and Drug Administration. https://www.fda.gov/files/drugs/published/N20571S023-Irinotecan-Clinical-BPCA.pdf

29. Vaidyanathan, J. 2008. Clinical pharmacology review for Anastrozole (Arimidex). NDA22214. Feb. 2008. Silver Spring, MD: Food and Drug Administration. https://www.fda.gov/media/75522/download

30. Cohen, M. 2003. Medical review for Fludarabine Phosphate. NDA 20038. July 2003. Silver Spring, MD: Food and Drug Administration. https://www.fda.gov/files/drugs/published/N20038S028-Fludarabine-Clinical-BPCA.pdf

31. https://www.fda.gov/drugs/development-resources/approved-active-moieties-have-appeared-nationalinstitutes-healths-nih-annual-priority-list-which

32. Pediatric Exclusivity Determinations List. Silver Spring, MD: Food and Drug Administration. https://www.fda.gov/media/132652/download

33. Administrative and Correspondence document. NDA 205582. https://www.accessdata.fda.gov/drugsatfda_docs/nda/2014/205582Orig1s000AdminCorres.pdf

34. FDA written requests issued. Silver Spring, MD: Food and Drug Administration. https://www.fda.gov/drugs/development-resources/written-requests-issued

35. RACE. Retrieved from https://www.congress.gov/bill/114th-congress/senate-bill/3239

36. Barone A, Casey D, McKee A, Reaman G. Cancer Drugs approved for use in children: Impact of legislative initiatives and future opportunities. Pediatric Blood and Cancer. 2019: 66(8): e27809

37. Ye J, Reaman G, De Claro RA, Sridhara R. A Bayesian approach in design and analysis of pediatric cancer clinical trials. Pharmaceutical Statistics. 2020: doi.org/10.1002/pst.2039

\section{Hosted file}

TABLE1.docx available at https://authorea.com/users/324759/articles/481458-a-review-of-theexperience-with-pediatric-written-requests-issued-for-oncology-drug-products

\section{Hosted file}

TABLE2.docx available at https://authorea.com/users/324759/articles/481458-a-review-of-theexperience-with-pediatric-written-requests-issued-for-oncology-drug-products

\section{Hosted file}

TABLE3.docx available at https://authorea.com/users/324759/articles/481458-a-review-of-theexperience-with-pediatric-written-requests-issued-for-oncology-drug-products

\section{Hosted file}

TABLE4.docx available at https://authorea.com/users/324759/articles/481458-a-review-of-theexperience-with-pediatric-written-requests-issued-for-oncology-drug-products 


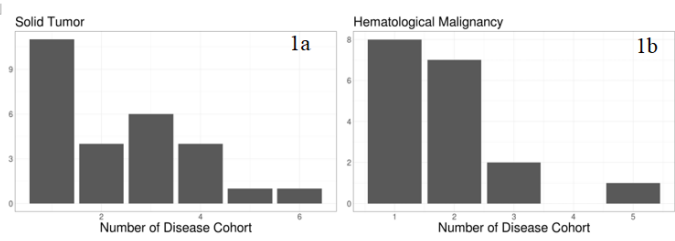

